\title{
An investigation of dolphin neocortex using computed tomography and magnetic resonance imaging
}

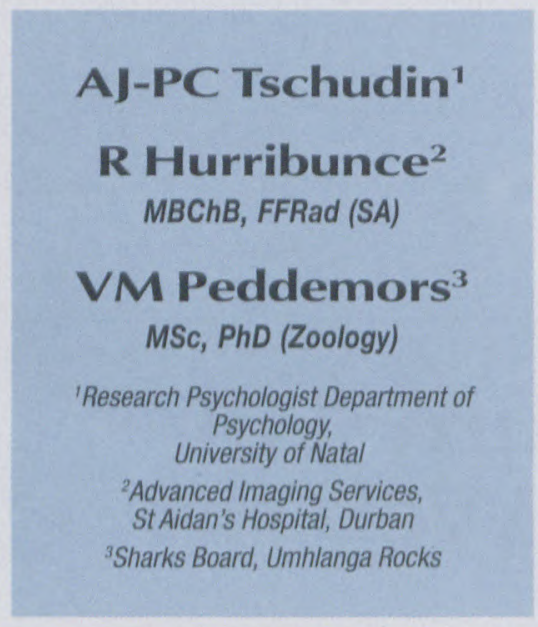

\section{Abstract}

The value of the conventional medical imaging applications of Computed Tomography (CT) and Magnetic Resonance Imaging (MRI) to areas such as medical diagnosis and surgical planning are widely documented; however, less conventional uses also exist for such technology. This paper documents an example of the diverse, interdisciplinary value of neuroradiological techniques such as CT and MRI.

An assumption has been made, in the absence of data for dolphins, that the uniquely high relative neocortex volume in primates is responsible for setting them apart from all other orders of mammals, in terms of social intelligence. An investigation of dolphin brain structure was undertaken to verify or dismiss this assertion using three species of differing social structure: solitary humpback (Sousa chinensis), social bottlenose (Tursiops truncatus) and gregarious common (Delphinus delphis) dolphins.

CT provided good quality images for calculation of cranial volume but only permitted gross imaging of the supra- and infratentorial regions. MRI provided greater differentiation of the different brain regions, thereby allowing volumetric measurements of the neocortical region for all subjects. There was no significant difference in neocortex calculations from fresh and frozen material, suggesting that this technique may be used on rare specimens which are frozen before examination by

biologists. Correlation of neocortical development with group size was significant $(\mathrm{p}<0.001)$.

\section{Introduction}

The recent and rapid advancement in technology has extended the boundaries of science to new frontiers, not remotely anticipated by the pioneers of our diverse disciplines. If one had proposed, twenty years ago, that a dolphin head was to be X-rayed, that would have drawn some interest. But to suggest the use of computed tomography to study dolphin neuroanatomy, without even making mention of a technique involving an emitted radio frequency within a high-powered magnet, could quite possibly have diminished one's scientific repute. However, in effect, the procedures of CT and MRI have made this interdisciplinary study possible and enhanced the research benefits available through modern neuroimaging technology.

to page 33 


\section{An investigation of dolphin neocortex}

using CT and MRI

\section{from page 32}

The study was conceptualised after a reading of the literature on relative brain size in mammals. Herein, it was asserted that the human primate was the only animal in the order of primates with an exceptional brain size. ${ }^{1-2}$ Additionally, primates were supposedly "brainier", in terms of relative neocortex volume, than all the other orders of mammals based on the relationship between social complexity and neocortex in primates. ${ }^{3-5}$ However, it appeared as if dolphins and other marine mammals, including whales and porpoises (comprising the Order Cetacea), had been omitted from consideration. This was surprising, as certain cetacean species are considered to have the largest absolute brain size of all animals, along with elephants. ${ }^{6}$ In addition, some dolphin species have the most convoluted brains and the largest cortical surface area of all animals, which further fuelled our hypothesis that dolphins may possess higher amounts of neocortex than primates, possibly dispelling the notion that social intellect is confined to primates. The measurement of dolphin neocortex therefore required accurate in situ measurement.

Incidentally caught dolphins, from the shark nets permanently set to protect bathers along the coast of KwaZulu Natal, provided ideal subjects for this study as they are retrieved fresh from the nets and dissected. In some instances, the subjects used were obtained from remote locations and/or could not be scanned while fresh, prompting the question of whether frozen and defrosted material would yield results of the same quality as if scanned while fresh. If defrosted material could provide data of the same accuracy as fresh specimens, there could be a potential benefit to animal researchers working with rare species in isolated locations as many cetacean researchers only obtain material from stranded animals originating from distant locations, which is usually frozen before examination. To ascertain the usefulness of such material for future international studies, fresh brain material was compared with that of frozen and defrosted animals.

\section{Methodology}

Dolphin skeletal material is valuable for taxonomic and morphometric research, hence skulls cannot be damaged for access to the brain. As the focus of this study was on dolphin neocortex, a suitable technique requiring no manual dissection or histological analysis was necessary. As estimates of cranial volume, obtained by volume measurements of the skulls, do not supply reliable measurements of neocortex, in the absence of actual data, the study appeared highly difficult to execute. The method of CT was initially suggested, after an attempt at its application in another research effort by the Sharks Board and the Delft University of Technology, Netherlands, into the investigation of the dolphin sonar-producing structures. The Department of Radiology, University of Natal Medical School, was consulted and gave permission for the use of its neuroimaging facilities, in addition to facilitating liaison with the private practice of Advanced Imaging Services, for the use of MRI.

\section{Subjects}

The dolphins forming the subjects for this study had all asphyxiated in the antishark nets set along the KwaZulu Natal coast. Specimens were obtained from the Sharks Board, following their measurement and dissection. The subjects were all sexually mature animals, representing three different dolphin species that exhibit various levels of social structure, including solitary humpback dolphins (Sousa chinensis), social bottlenose dolphins (Tursiops truncatus) and gregarious common dolphins (Delphinus delphis). Following the scanning of fresh subjects, the material was placed in the Sharks Board freezer at $-20^{\circ} \mathrm{C}$. When required for subsequent re-analysis, frozen material usually took between 48 to 72 hours to defrost.

\section{Apparatus}

The Picker PQ2000 CT Scanner at Wentworth Hospital was the first instrument used for the study. Although CT provided crucial data on cranial volume, it only permitted gross imaging of the supra- and infra-tentorial regions (Figure 1). MRI was therefore suggested as a more appropriate technique for volumetric measurement, as it provides greater differentiation of the different brain regions, allowing discrimination

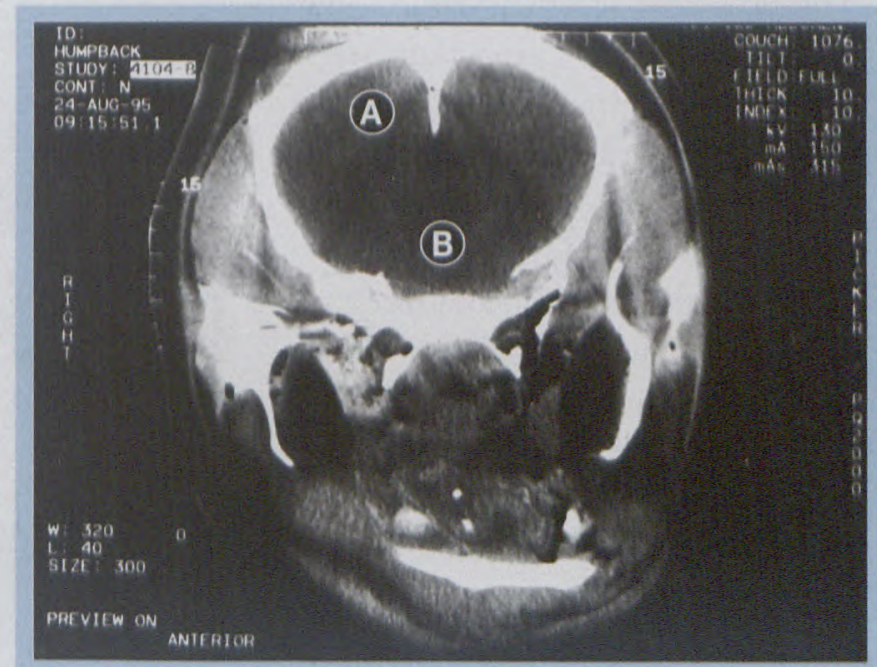

Figure 1: CT image of a dolphin brain showing differentiation between (A) supra- and (B) infra-tentorial regions. 


\section{from page 33}

between the neocortex (defined as cerebral cortex) and rest of the brain (brainstem and cerebellum) (Figure 2), thereby furnishing a more accurate analytical tool than CT. Subsequent MRI scans were made using a Siemens Magnetom Impact (1 Tesla Unit) MRI Scanner (Advanced Imaging Services - AIS), and the General Electric (1.5 Tesla Unit) Signa Scanner (Wentworth Hospital).

\section{Procedure}

Following dissection of the subjects, the heads were removed and sealed in plastic protective bags to prevent any possible contamination. The subjects were transported to the scanning unit in a cooler box and scanned in the coronal (human axial) plane using a slice thickness of $6 \mathrm{~mm}$ and interslice distance of $6 \mathrm{~mm}$ for MRI and $10 \mathrm{~mm}$ thick slices with an inter-slice gap of $10 \mathrm{~mm}$ for CT. The subject was scanned in a prone, "tailfirst" position for CT and MRI at AIS, while a "head-first" position was adopted for scanning at Wentworth.

Images from CT were stored on tape, while those from MRI were recorded on optical disc and hard drive. The neocortex (cerebral cortex), the brainstem and the cerebellum were subsequently trackballed using a digitiser. The area of the regions of interest on each slice was calculated. All slice areas were added, taking into account slice thickness and interslice gap, to arrive at a measurement of volume for each region.

To ascertain whether or not defrosted material could be reliably used to obtain neocortical volume, several specimens were scanned while fresh and subsequently scanned while frozen and defrosted, according to the above procedure.

\section{Results}

It was evident from visual examination of CT and MRI (Figures 1 and 2) that MRI provided a better image

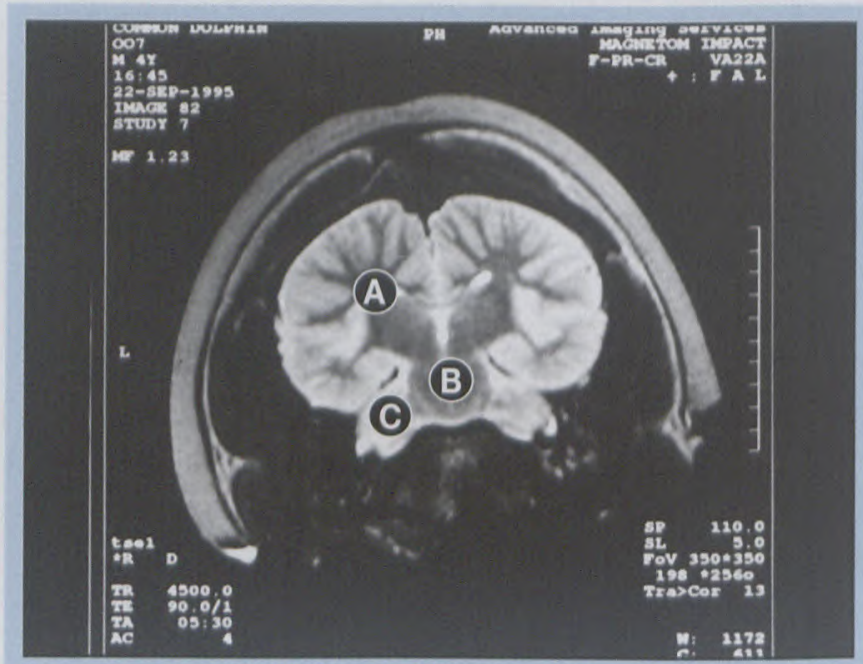

Figure 2: MRI image of a dolphin brain, displaying neocortex A - cerebral cortex, B - brainstem and C - cerebellum.

for volumetric analysis of different cortical regions than CT. However, it was not as easy to determine whether or not non-fresh material (frozen and/or defrosted) could be used in volumetric research using MRI, as no documented research could be located in this regard. An example of a subject scanned under the three different states (fresh, frozen and defrosted) is presented in Figure 3. While the fresh specimen provided the most defined

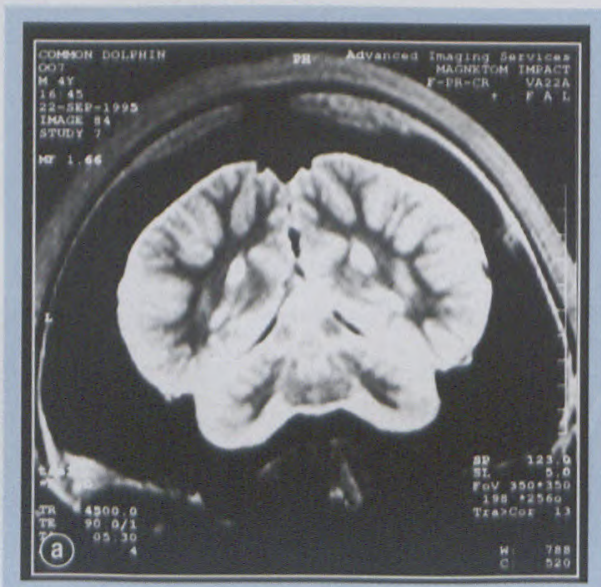

Figure 3: MRI scans of dolphin brain material under various conditions. From a to $\mathrm{c}$ : fresh, frozen and defrosted. image, the differentiation of material in the image of a defrosted brain was sufficiently clear to allow calculation of brain volumes. Through the application of a Wilcoxon test, it was established that no significant difference existed between the neocortex ratio as determined by fresh and defrosted material $(p>0.1)$. Frozen material could not be analysed as no signal was provided.

For the calculation of neocortex ratios, only the MRI measurements of neocortex volumes were used, as CT overestimated values. The high dolphin neocortex

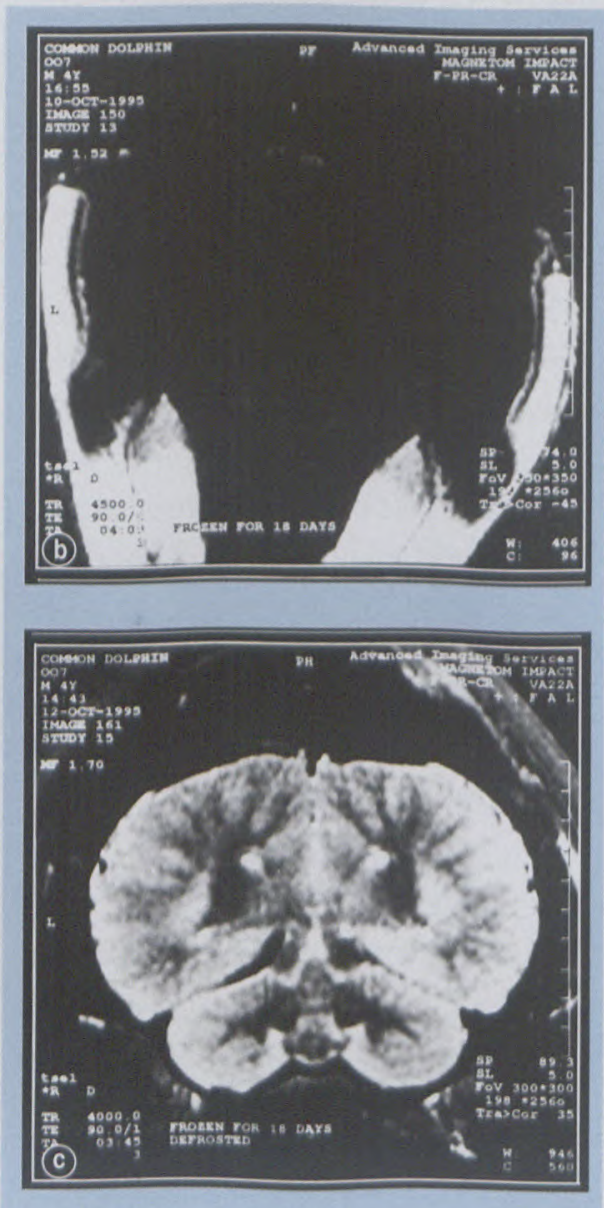

to page 35 


\section{from page 34}

ratios (ratio of cerebral cortex to rest of the brain) were significantly correlated with sociality, as evidenced by the group size of the species $(\mathrm{p}<0.001) .{ }^{8}$ Comparison to values for all primates, including humans, ${ }^{4}$ highlighted the elevated ratio values for the dolphins studied (Figure 4 - from reference 8).

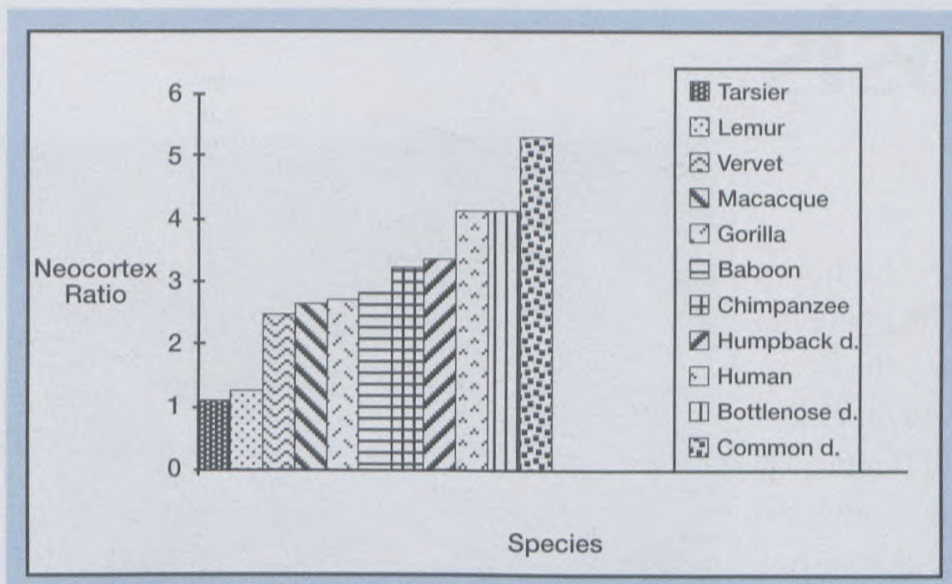

Figure 4: Dolphin neocortex ratios in comparison to primate neocortex ratios. (Primate data from reference 4, dolphin data from reference 8).

\section{Discussion}

The use of CT and MRI have rarely been documented in comparative research; however, these techniques have been utilised in anatomical studies, specifically those associated with delphinid bioacoustics. ${ }^{9,10}$ Additionally, fundamental comparative brain research has the potential to benefit greatly from the successful application of CT and MRI as is evidenced by the ability of our pilot study to measure dolphin neocortex using neuroimaging, a feat which would otherwise have remained beyond our capabilities. The finding that specimens can be frozen, stored, defrosted and subsequently analysed with reliable results indicates that rare species, from isolated or technologically undeveloped areas can still be examined. The result of most conse- quence from this study is that some dolphin species have larger relative neocortices than humans and that all the dolphin species examined had higher neocortex ratios than the human's closest relative, the chimpanzee. ${ }^{8}$ This is of interest, as it calls for a re-conceptualisation of mammalian brain evolution. The strong relationship between social complexity and neocortex in dolphins also challenges the assumption that social intelligence is distinctive of the order of primates. ${ }^{8}$ Such studies can only support the proposal that the utility of such technology lies in extending the accuracy and boundaries of scientific investigation, making this more accessible to interdisciplinary research.

\section{Acknowledgements}

We are deeply indebted to the two institutes which allowed us free access to their scanners, without which this project would not have been feasible. Our sincere thanks are expressed to the radiographers and partners at Advanced Imaging Services, St Aidan's Hospital, Durban, the staff at Wentworth Hospital and the Department of Radiology, University of $\mathrm{Na}$ tal Medical School. The Director and staff of the Sharks Board are also thanked for their assistance with this project.

\section{References}

1. Martin RD. Relative brain size and basa metabolic rate in terrestrial vertebrates. Nature, London 1981; 293: 57-60.

2. Martin RD. Primate Origins and Evolution: A phylogenetic reconstruction. London: Chapman \& Hall. 1990.

3. Sawaguchi T, Kudo H, Neocortical development and social structure in primates. Primates 1990; 31: 283-290.

4. Dunbar RIM. Neocortex size as a constraint on group size in primates. Journal of Human Evolution, 1992; 20: 469-493.

5. Dunbar RIM. Neocortex size and group size in primates: a test of the hypothesis, Journal Of Human Evolution, 1995; 28: 287-296.

6. Ridgway SH. Dolphin Brain Size. In: Bryden MM, Harrison R. Research on Dolphins. New York: Oxford University Press. 1986; 59 70

7. Elias H, Schwartz D. Surface areas of the cerebral cortex of mammals determined by stereological methods. Science, 1969; 166 11]-13.

8. Tschudin A, Daji K, Moomal Z, Peddemors V, Royston D. Neocortex Size and Group Size in Dolphins. South African Journal of Science (submitted)

9. Cranford TW. The anatomy of acoustic structures in the spinner dolphin forehead as shown by $\mathrm{x}$-ray computed tomography and computer graphics. In: Nachtigall PEN Moore PWB. Animal Sonar Processes and Performance. New York: Plenum Press. 1988.

10. Brouwers MEL, Kaminga C, Klooswijk AIJ Terry, RP. The use of computed tomography in cetacean research. Airsac determination of Lagenorhynchus albirostris Part 1. Aquatic Mammals, 1990;16(3):145-55. 